\title{
Total Multimode Reflection at Facets of Planar High-Contrast Optical Waveguides
}

\author{
Manfred Hammer and E. van Groesen
}

\begin{abstract}
Based on rigorous mode expansion simulations, we consider the problem of guided light reflection at rectangular end facets of planar dielectric waveguides. Emphasis is on multimode structures with high refractive index contrast and on configurations that show a high reflectivity. Given the matrix of reflection coefficients for the guided fields, one can compute the maximum power reflectance for an incident mode superposition. While even for a substantial refractive index contrast across the facet the maximum reflected power for a single incoming mode is always moderate, the reflectivity may rise to a level that justifies the attribute total if one considers specific superpositions of at least two guided modes. The paper shows the results of numerical experiments for two series of symmetrical and nonsymmetrical waveguides and identifies conditions on the facet geometry and the exciting field, which are prerequisites for the full reflection effect.
\end{abstract}

Index Terms-Dielectric waveguide facet, integrated optics, microresonator cavities, numerical modeling, total reflection.

\section{INTRODUCTION}

A T THE END facet of a dielectric waveguide, an incoming confined lightwave is partly transmitted and partly reflected, where the guided backward traveling fraction of the optical power depends strongly on the waveguide geometry and the refractive index contrast. Although the facet problem has been widely investigated [1] in various settings, with techniques either of a more semianalytical [2]-[8] or a more rigorous numerical nature [9], [10], these studies mostly focus on minimizing the loss of optical power to radiation and reflection at the transition from the waveguide to the adjacent homogeneous space. Frequently coated [4], [6], [7] and/or angled [2], [5] facets are simulated and optimized for extremely low reflectivity.

So far, apparently only minor attention has been paid to configurations where the ended waveguide supports more than one mode per polarization and where the interest is in highly reflecting structures. As we discuss in Section IV, these two features are closely related: the relative reflected guided power can reach a level very close to unity for multimode input fields with specific relative amplitudes. These observations provide at least a starting point to a quantitative understanding of the pronounced resonance phenomena in some recently discussed microresonator devices [11], [12] where segments of such wide high-contrast waveguides serve as cavities.

Manuscript received January 16, 2002; revised April 30, 2002.

M. Hammer is with the Faculty of Mathematical Sciences, University of Twente, 7500 AE Enschede, The Netherlands (e-mail: m.hammer@math.utwente.nl).

E. van Groesen is with the MESA Research Institute, University of Twente, 7500 AE Enschede, The Netherlands.

Digital Object Identifier 10.1109/JLT.2002.800302
The investigation of multimode waveguide facets in this paper relies on a bidirectional mode expansion technique as described in [1] and [13]. Section II merely introduces the notation; details of the present implementation can be found in [12] and [14]. The subsequent reasoning is then based on the numerically computed matrix of reflection coefficients. The reflection levels observed for single-mode excitations in Section III change drastically if one sends in at least two guided fields simultaneously. This is the subject of Section IV, where we try to illustrate the underlying interference mechanism. The section includes detailed multimode reflectivity curves as well as a few examples of characteristic field patterns. While the previous sections are concerned with symmetric waveguides, Section V assesses the influence of a nonsymmetric layering.

\section{Multimode Facet Reflectivity}

Fig. 1 introduces the facet geometry and the relevant setting of coordinates. We restrict the problem to two spatial dimensions and to TE polarization, where only the single component $\mathcal{E}_{y}$ of the electric field is present. Where no other values are given, the simulations in this paper are meant for symmetric waveguides with a substrate and cover refractive index $n_{s}=n_{c}=1.45$, a refractive index $n_{a}=1.0$ of the homogeneous half space beyond the facet, and for a constant angular frequency $\omega=k c=$ $2 \pi c / \lambda$, given in terms of the vacuum wavenumber $k$, vacuum speed of light $c$, and the vacuum wavelength $\lambda=1.55 \mu \mathrm{m}$.

In common complex notation, the guided part of the optical electrical field at time $\tau$ in the waveguide region $z<0$ can be written as

$$
\begin{aligned}
\mathcal{E}_{y}(x, z, \tau)= & \sum_{m} a_{m} \frac{1}{\sqrt{P_{m}}} E_{y, m}(x) \exp \left(i \omega \tau-i \beta_{m} z\right) \\
& +\sum_{m}\left(\sum_{j} r_{m j} a_{j}\right) \\
& \times \frac{1}{\sqrt{P_{m}}} E_{y, m}(x) \exp \left(i \omega \tau+i \beta_{m} z\right)
\end{aligned}
$$

where the first term represents the incident field, the second term is the reflected part [(1) is not intended to cover the total electric field; see the remarks in the next paragraph], and $E_{y, m}$ and $\beta_{m}$ are the real planar guided mode profiles and the corresponding positive propagation constants. With each mode, a normalizing power $P_{m}$ is associated such that $P_{\mathrm{in}}=\sum_{m}\left|a_{m}\right|^{2}$ is the optical input power for amplitudes $a_{m}$ of the incident modes. Then, the total relative guided reflected power $R$ reads as

$$
R=\frac{1}{P_{\text {in }}} \sum_{m}\left|\sum_{j} r_{m j} a_{j}\right|^{2}=\frac{1}{P_{\text {in }}}|\mathrm{R} \boldsymbol{a}|^{2}=\frac{1}{P_{\text {in }}} \boldsymbol{a}^{\dagger} \mathrm{R}^{\dagger} \mathrm{R} \boldsymbol{a}
$$




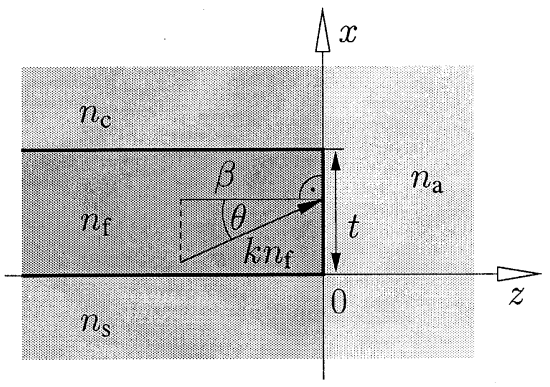

Fig. 1. The waveguide facet. $z$ and $x$ denote the Cartesian coordinate axes parallel and perpendicular to the film plane. The geometry is specified in terms of the film thickness $t$ and the refractive indices $n_{s}, n_{f}, n_{c}$, and $n_{a}$ of the substrate, the film, the cladding material, and the medium adjacent to the facet. A mode angle $\theta$ is assigned to each guided field with propagation constant $\beta$.

where $\boldsymbol{a}=\left(a_{j}\right)$ collects the input mode amplitudes, $\mathbf{R}=\left(r_{m j}\right)$ is the matrix of reflection coefficients of the facet for guided fields, and ${ }^{\dagger}$ denotes the adjoint. Besides $R$ and its restrictions to smaller mode sets, individual mode reflectivities will play a role below: if only mode $m$ has a nonvanishing input amplitude, the relative power reflected into that particular mode is directly $\left|r_{m m}\right|^{2}$. Motivated by the prospective relevance of these considerations for the formation of optical cavities, in this paper the term "reflectivity" is always used to indicate the relative optical power that is received by the backward traveling versions of a specific input mode set.

The following discussion assumes that the reflectivity matrix $\mathrm{R}$ is accessible for each facet, computed numerically with sufficient accuracy. For this purpose, we employed rigorous bidirectional eigenmode propagation simulations as formulated in [12] and [14]. The continuous part of the mode spectrum on the longitudinally homogeneous segments $z<0$ and $z>0$ is discretized by placing artificial boundaries suitably far apart from the waveguide core, where $\mathcal{E}_{y}$ is required to vanish. Separately, on both sides of the junctions, the optical field is expanded into series like (1), including a fixed number of forward and backward traveling, propagating, and evanescent terms. Then, bidirectional projection onto the elements of the modal basis sets yields a linear relation between the expansion coefficients on the adjacent segments; $R$ is the restriction to the confined fields of the waveguide segment. Hence, the transmitted and backward radiated, nonguided parts of the optical field are taken fully into account, although they do not show up explicitely in (1).

All simulations below use a $16-\mu \mathrm{m}$ computational window, centered around the symmetry plane $x=t / 2$, and the 115 lowest order expansion terms on both segments. While the hard boundary conditions are not undisputed in the present context [1], [8], [10], at least on the scale of Figs. 3-8, we found the results to be converged with respect to the width of the computational window and with respect to the number of expansion terms. More specifically, varying the width of the domain in the range between 14 and $18 \mu \mathrm{m}$ and adjusting independently the number of mode basis elements between 100 and 130 produced hardly any visible effects on the reflectivity levels displayed e.g., in Fig. 5. Apparently, the computational window is sufficiently wide to sufficiently suppress the oscillatory behavior of the simulation results as observed for specific configurations in [1], [8], and [10]. Being interested in reflectivity levels on a scale between 0 and 1 with an indeterminacy of about one percent only, we may thus disregard the influence of the artificial boundary conditions for the problems considered in this paper. Note that the computational window is substantially larger than the $x$ intervals shown in Figs. 4, 7, and 9; perturbations of the field in the displayed domains by waves that originate from the facet and are reflected by the artificial boundaries can be expected to be insignificant.

\section{SINGLE-Mode INPUT}

To supplement the numerical computations, occasionally a reasoning based on the ray model for guided fields is helpful. For each mode $m$, an angle $\theta_{m}$ is defined via the relation $\cos \theta_{m}=\beta_{m} /\left(k n_{f}\right)$. This angle may be interpreted alternatively as the angle of incidence at the facet of the plane waves that constitute the guided field in the core (see Fig. 1).

In the framework of the ray model, one might then expect that an input mode encounters a high reflection if its mode angle exceeds the critical angle $\theta_{\text {crit }}$ for total reflection at the facet interface, given by $\sin \theta_{\text {crit }}=n_{a} / n_{f}$. According to Fig. 2 , the condition $\beta / k<\left(n_{f}^{2}-n_{a}^{2}\right)^{1 / 2}$ is satisfied for the high-order modes in the structures of this paper. The requirement $\beta>k n_{s}$ for guided fields in the core imposes an upper limit $\theta_{\max }$ to the mode angles, where $\sin \theta_{\max }=\left(1-n_{s}^{2} / n_{f}^{2}\right)^{1 / 2}$. Consequently, only facet configurations with

$$
n_{f}^{2}>n_{s}^{2}+n_{a}^{2}
$$

may be subject to a pronounced reflectivity.

Fig. 3 gives some evidence for this simplified reasoning. The two waveguides support modes up to order 8(a) and 12(b). In both cases, the four lowest order fields have mode angles below the critical values $\theta_{\text {crit }}$, and the reflectivity remains small. For the higher order modes above the level $\theta_{\text {crit }}$, the single-mode reflectivity is significantly higher, with a smooth transition at $\theta_{\text {crit }}$. Nevertheless, large amounts of power still pass the facet, are radiated, or are reflected into other modes. The plane waves that form the field in the core are bounded with a certain amount of the mode power traveling in the substrate and cover regions; hence, the ray model fails in this respect.

The first two columns of Fig. 4 illustrate the field patterns in the structures of Fig. 3, when single high-order modes are launched. Apart from small contributions from other modes, the checkerboard-like pattern in the core region is constituted by the forward and backward versions of the input mode with different amplitudes; one observes a partly standing, partly traveling field in the core.

For single-mode input, the radiated field in the homogeneous region $z>0$ appears as a superposition of two cylindrical waves, originating from the edges of the core at the facet, where the cylindrical form is disturbed by the facet discontinuity in the substrate and cover regions. Apart from the edges, almost no traveling fields seem to traverse the facet plane in the region $0<x<t$, where similar to the film/substrate and film/cover interfaces only exponential tails are visible. Here the ray model is correct. 

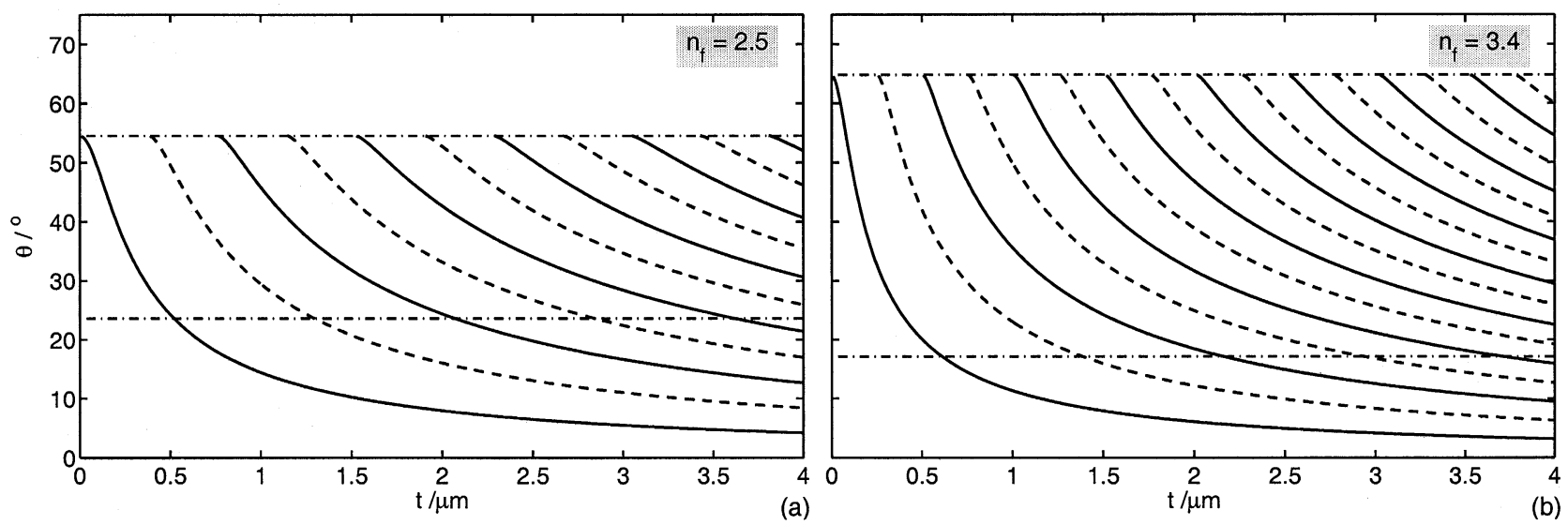

Fig. 2. Angles $\theta$ associated with guided mode propagation constants versus the waveguide thickness for symmetrical planar waveguides as specified by Fig. 1 with core refractive indices (a) $n_{f}=2.5$ and (b) $n_{f}=3.4$. See the text for other parameter values. The lower horizontal lines indicate the critical incidence angles $\theta_{\text {crit }}=23.6^{\circ}$ (a) and $\theta_{\text {crit }}=17.1^{\circ}$ (b) for total plane-wave reflection at an interface between two media with refractive indices $n_{f}$ and $n_{a}$. The upper lines are placed at the cutoff angles $\theta_{\max }=54.5^{\circ}$ (a) and $\theta_{\max }=64.8^{\circ}(\mathrm{b})$ for guided wave propagation in the ended waveguides.
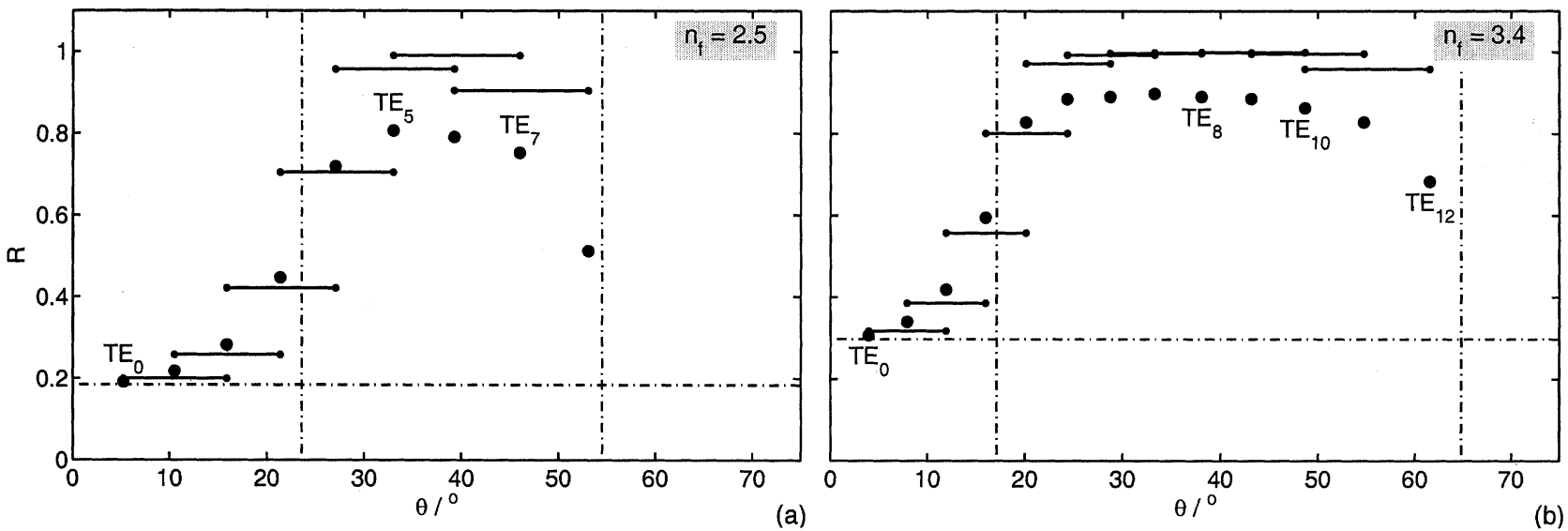

Fig. 3. Modal reflectivities $R$ versus the (discrete) mode angle, for a facet according to Fig. 1 with waveguide thickness $t=3.15 \mu \mathrm{m}$ and film refractive indices (a) $n_{f}=2.5$ and (b) $n_{f}=3.4$. Dots denote the relative guided power $\left|r_{m m}\right|^{2}$ that is reflected if a single guided field with the proper mode angle is launched. Horizontal bars mark the guided reflection if the facet is excited by a superposition (4) of the two modes with angles at the bar end points. The vertical lines correspond to the critical incidence angles $\theta_{\text {crit }}$ and the maximum mode angles $\theta_{\max }$, as given in the caption of Fig. 2 .

Fig. 5 displays further results for the reflection of single input modes, with reflectivity values as a function of waveguide thickness. The shape of these curves is found to be quite uniform for all modes. At small thicknesses, close to cutoff, the almost flat mode profile spreads wide into the cover and substrate regions. Hence, the curves start at a level that is given by the plane-wave reflectivity for perpendicular incidence on an interface between the substrate and background materials. With growing $t$, the modes become more and more confined in the core region with a still large mode angle, resulting in an increase of the reflectivity. For very thick films, the field strengths at the upper and lower core interfaces become negligible, while the mode angles and consequently the $x$ derivatives of the mode profiles tend to zero. This is again the situation of perpendicular plane-wave incidence on a dielectric interface, now with the higher refractive index contrast $n_{f} \mid n_{a}$. All reflectivities approach the corresponding level for large $t$ asymptotically according to the Fresnel formula for perpendicular polarization, with the mode angle used as the incidence angle.

If one accepts a guided-wave viewpoint for the resonator devices considered in [11] and [12], the moderate single-mode reflectivities of Figs. 3-5 are not sufficient to explain the quality of some of the resonances that are observed in the rectangular cavity waveguide segments. While some of the minor resonances indeed show single-mode standing waves, the most pronounced resonances always include more than one modal basis field (cf. the plots in [12]). This led to the investigation of the reflection phenomena related to multimode excitations in Section IV.

\section{Multimode Excitations}

Aiming at specific input fields with high reflectivity, one could try to suppress the sources of transmission and radiation that are visible in the left and center plots of Fig. 4. The cylindrical waves should vanish if one launches two modes simultaneously such that the total electric field is zero at the facet edges. This will only be possible for fields that share the same symmetry; Fig. 6 shows examples.

Assume that indexes 1 and 2 are assigned to these two modes, with the input amplitudes $a_{1}$ and $a_{2}=a a_{1}$ connected by the rel- 

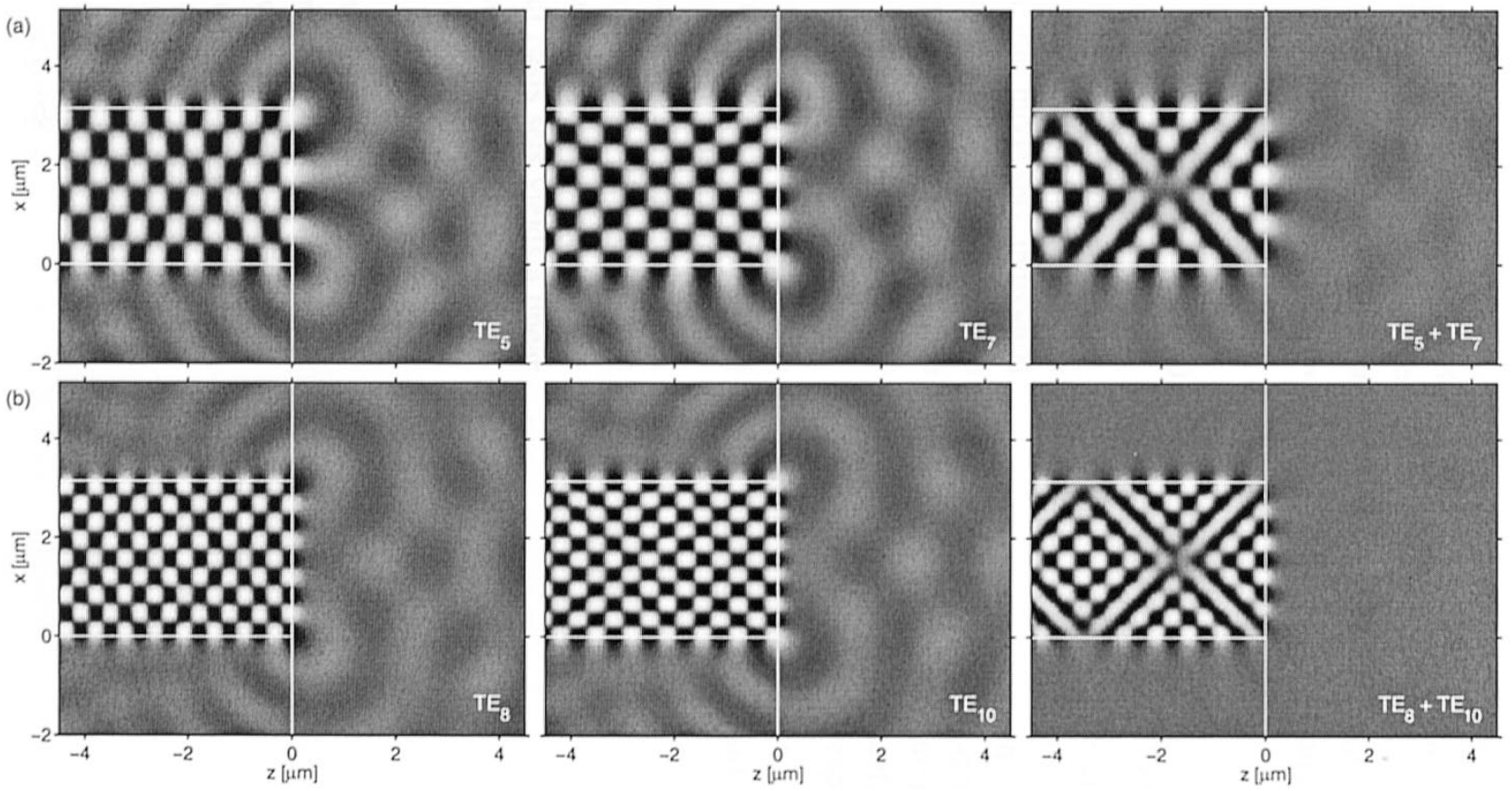

Fig. 4. Snapshots of the electric field $\mathcal{E}_{y}$ at selected times for facets as sketched in Fig. $1, t=3.15 \mu \mathrm{m}, n_{f}=2.5$ (a, top row) and $n_{f}=3.4$ (b, bottom row). For the lower index core (a), the fifth- and seventh-order modes are first launched separately (left and center), then simultaneously with a specific relative amplitude according to (4) (right). For the higher index film (b), the eighth- and tenth-order modes and their superposition were considered. White and black regions mean positive and negative field strength, and the gray background (the surrounding in the lower right inset) indicates the zero level. The reflectivities corresponding to these plots are (a) $\left|r_{55}\right|^{2}=0.81,\left|r_{77}\right|^{2}=0.75, R_{2}>0.990$ and (b) $\left|r_{88}\right|^{2}=0.89,\left|r_{1010}\right|^{2}=0.86, R_{2}>0.998$.
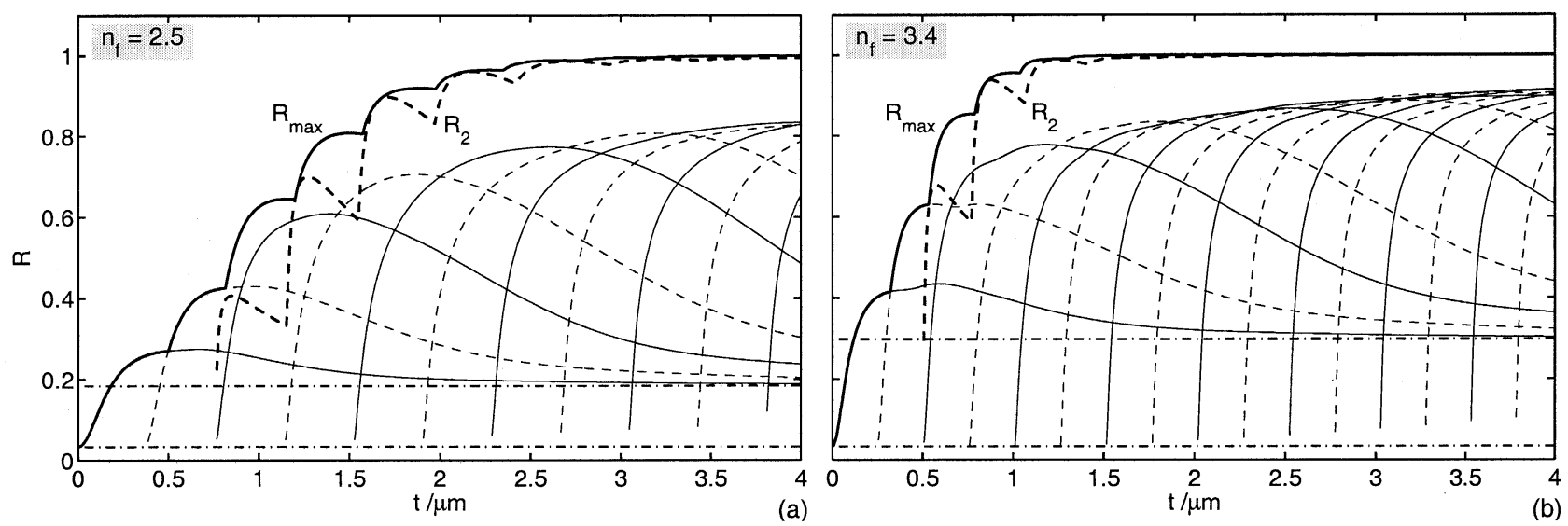

Fig. 5. Mode reflectivities $R$ versus the film thickness $t$, for facet configurations according to Fig. 1 with film refractive indices (a) $n_{f}=2.5$ and (b) $n_{f}=3.4$. The thin curves indicate the reflectivities of individual modes, where continuous and dashed lines correspond to even and odd input fields, respectively. The thicker continuous curve marks the highest reflectivity level $R_{\max }$ that can be reached by any incoming guided field superposition, and the dashed line $R_{2}$ is meant for a bimodal input with relative amplitudes according to (4). Horizontal lines are the reflectivities predicted by the Fresnel equations for perpendicular incidence of plane waves on dielectric interfaces with refractive indices $n_{f} \mid n_{a}$ (upper line) and $n_{s} \mid n_{a}$ (lower line).

ative amplitude $a$. Then the total field $\mathcal{E}_{y}$ vanishes at the origin if

$$
a=-\frac{\left(1+r_{11}\right) \frac{E_{y, 1}(0)}{\sqrt{P_{1}}}+r_{21} \frac{E_{y, 2}(0)}{\sqrt{P_{2}}}}{r_{12} \frac{E_{y, 1}(0)}{\sqrt{P_{1}}}+\left(1+r_{22}\right) \frac{E_{y, 2}(0)}{\sqrt{P_{2}}}} .
$$

The mode symmetry implies $\mathcal{E}_{y}(t, 0, \tau)=0$. This choice of input amplitudes results in the two-mode reflectivity

$$
R_{2}=\frac{\left|r_{11}+r_{12} a\right|^{2}+\left|r_{21}+r_{22} a\right|^{2}}{1+|a|^{2}}
$$

For the two configurations of Fig. 4, we indeed obtained very high reflection levels of $R_{2}>0.99$, where the difference to unity is well below the accuracy limit that can be expected for the numerical simulations (although here the artificial boundaries should have only minor influence). The third column of Fig. 4 contains plots of the corresponding field patterns. One observes almost purely standing waves inside the core and evanescent tails outside.

The $R_{2}$ data of Fig. 5 proves that this is not a singular incidence. The curves show the maximum of the reflectivity over all pairs of modes at the particular thickness. Levels of almost one can be achieved, provided that at least two modes of equal 

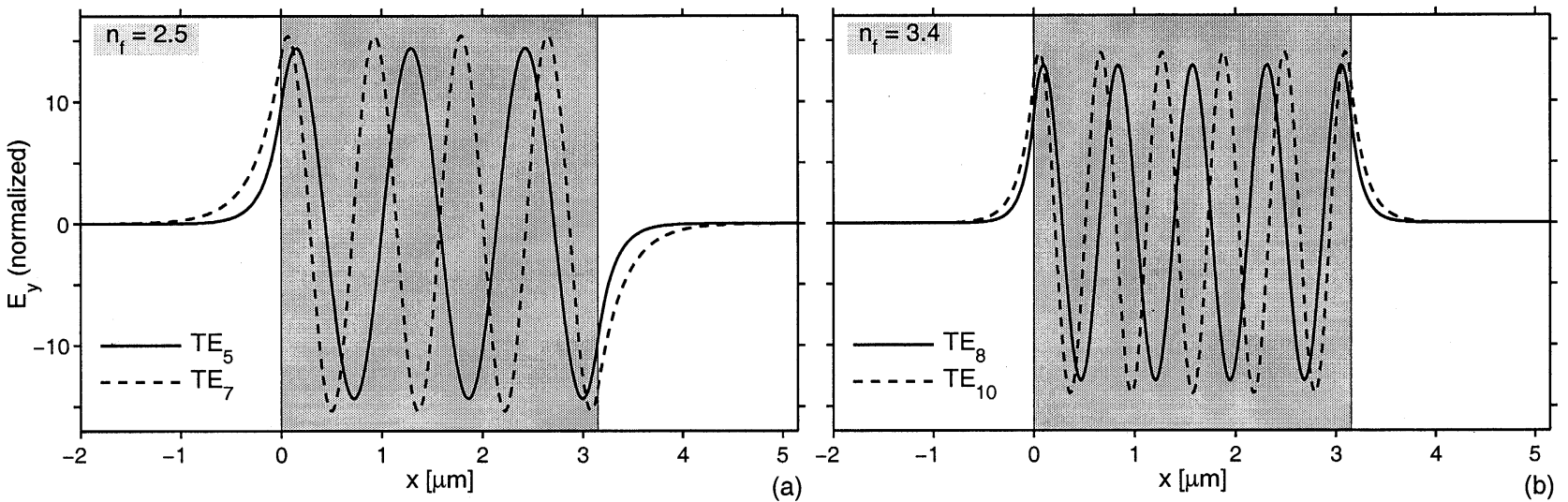

Fig. 6. Normalized guided mode profiles that constitute the basis for the high reflection configurations of Figs. 3 and 4 . The mode angles associated with these profiles are (a) $33.0^{\circ}\left(\mathrm{TE}_{5}\right)$ and $46.0^{\circ}\left(\mathrm{TE}_{7}\right)$ for the lower index core $n_{f}=2.5$ and (b) $38.1^{\circ}\left(\mathrm{TE}_{8}\right)$ and $48.7^{\circ}$ ( $\left.\mathrm{TE}_{10}\right)$ for the high contrast configuration $n_{f}=3.4$. The waveguide thickness is $t=3.15 \mu \mathrm{m}$ in both cases.
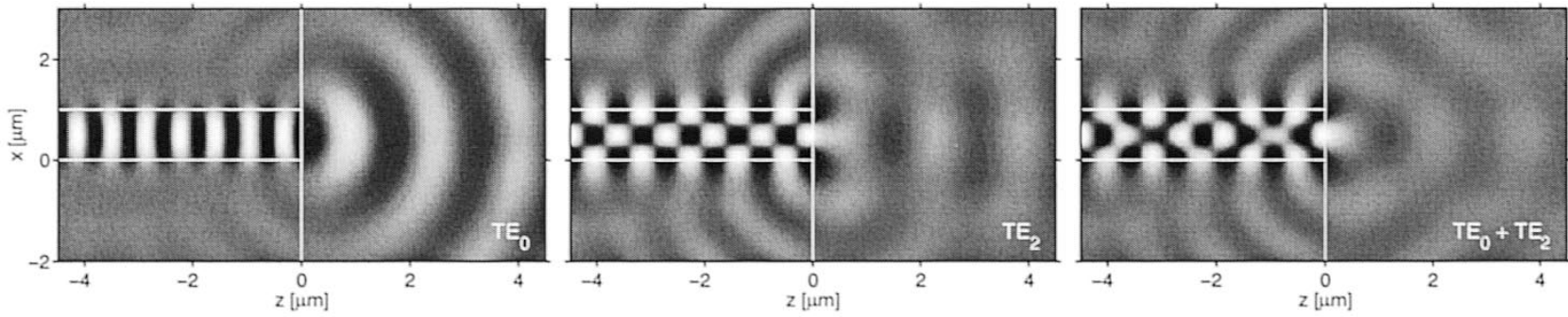

Fig. 7. Electric field patterns for a facet excitation by low-order modes: the fundamental field (left), the second-order mode (center), and the superpostion of the two that leads to the maximum guided reflected power (right). Here, the relative amplitude does not satisfy (4). The configuration is one of the structures of Fig. 5(a): $n_{f}=2.5, t=1.0 \mu \mathrm{m}$

symmetry exist with mode angles above the critical level $\theta_{\text {crit }}$ and with a sufficiently high single-mode reflectivity (compare Figs. 2 and 5).

The recipe (4) for a high reflectivity obviously fails for lower order modes at small waveguide thicknesses. Fig. 7 addresses such a configuration, where one of the above conditions is not satisfied. At $t=1.0 \mu \mathrm{m}$ in Fig. 5(a), the fundamental and secondorder modes make up the only mode pair with equal symmetry, where the $\mathrm{TE}_{0}$ mode angle is much smaller than $\theta_{\text {crit }}$. Significant traveling parts of the field cross the facet plane in the central core region for $\mathrm{TE}_{0}$ input, and there is no possibility for destructive interference with the cylindrical waves originating from the core edges for $\mathrm{TE}_{2}$ input. In this case, the field at the core center should be diminished to maximize the reflectivity. Consequently, the third inset of Fig. 7, a field snapshot for the $\mathrm{TE}_{0}+\mathrm{TE}_{2}$-superposition with the highest reflectivity, shows nonzero field amplitudes at the core edges. The reflectivity value would be below the single-mode level for the $\mathrm{TE}_{2}$ mode if the relative amplitude would have been according to (4) (see Fig. 5).

Finally, one might be interested in the maximum reflectivity value with respect to arbitrary superpositions of guided input fields. The task to find the normalized amplitude vector $\boldsymbol{a}$ with the largest relative reflected power (2) amounts to solving an eigenvalue problem $R^{\dagger} R \boldsymbol{a}=\rho \boldsymbol{a}$ for the reflectivity matrix, where the largest eigenvalue $\rho$ directly gives the maximum reflectivity $R_{\max }$. Fig. 5 includes the corresponding curves.

As long as only one or two modes are guided for thin waveguides, $R_{\max }$ necessarily coincides with the larger of the single-mode reflectivities. Just as $R_{2}$ does, the $R_{\max }$ curves exhibit kinks where new modes appear with growing $t$. As soon as two modes with equal symmetry come into play, $R_{\max }$ is significantly higher than the single-mode levels. With $t$ further increasing, the maximum reflectivity approaches unity more or less rapidly, depending on the refractive index contrast. Even in this asymptotic region, $R_{\max }$ is always slightly higher than the reflectivity $R_{2}$ for a bimodal input field: while the simple heuristic that led to (4) considers reflection into the input modes only, these two fields couple to other guided modes and to the radiation field. Those contributions to the field strength at the facet edges are not compensated for by (4). Nevertheless, the extreme levels in particular for the high-contrast configuration (b) for both $R_{2}$ and $R_{\max }$ seem to justify the term "total reflection" for this interference phenomenon.

\section{NONSYMMETRIC WAVEGUIDES}

Although the previous reasoning relies crucially on symmetry arguments, Figs. 8 and 9 show that the observations remain valid for moderately nonsymmetric waveguides. For Fig. 8, we consider facets with fixed large waveguide thicknesses that allow configurations with complete reflection in the symmetric setting $n_{s}=n_{c}$. With increasing substrate refractive index, the reflectivities $R_{\max }$ and $R_{2}$ first stay on their high level. A pronounced drop in the reflectivity occurs when the relevant highorder modes start to disappear due to the growing asymmetry. 

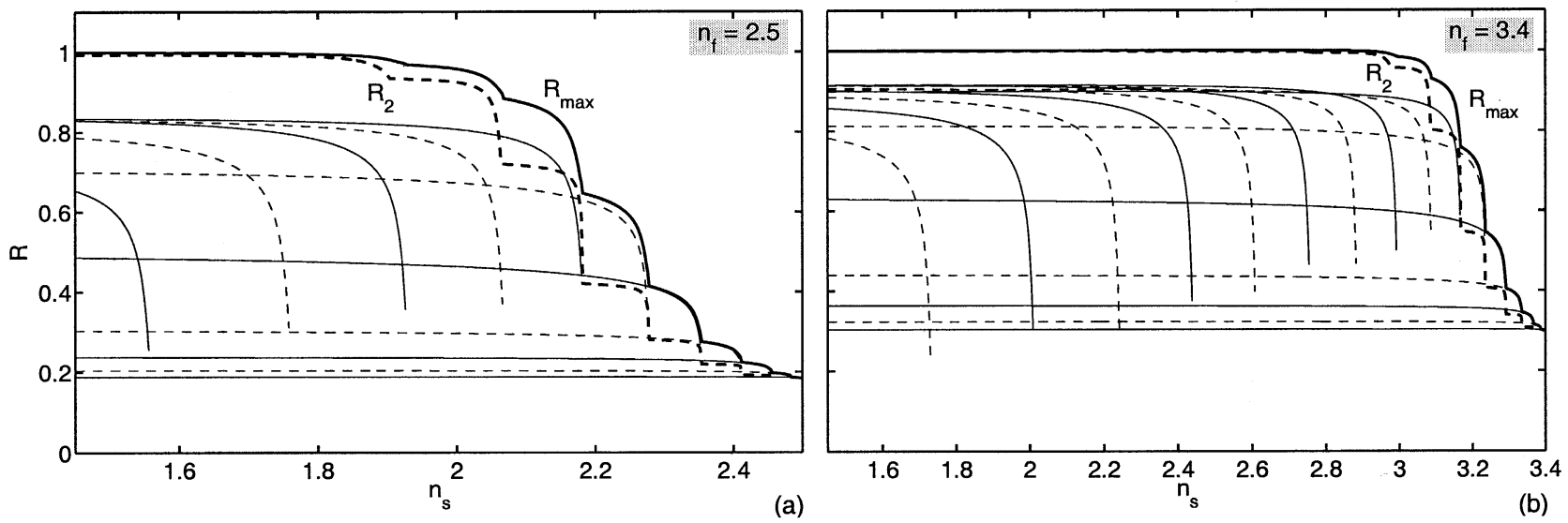

Fig. 8. Mode reflectivities $R$ versus the substrate refractive index $n_{s}$ for facets of nonsymmetric waveguides according to Fig. 1 with a core thickness $t=4.0 \mu \mathrm{m}$ and film refractive indices (a) $n_{f}=2.5$ and (b) $n_{f}=3.4$. See the caption of Fig. 5 for the interpretation of the line styles.
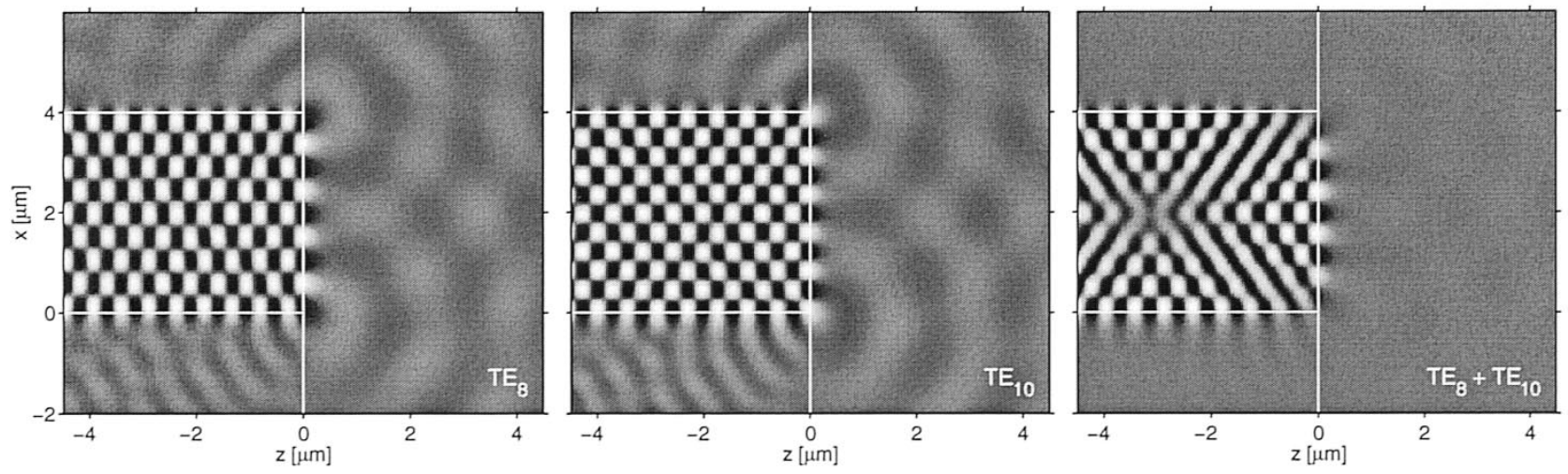

Fig. 9. Analogous to Fig. 4(b), for a nonsymmetric waveguide with thickness $t=4.0 \mu \mathrm{m}$, film refractive index $n_{f}=3.4 \mu \mathrm{m}$, and a substrate refractive index of $n_{s}=2.4$. The reflectivity values observed in the three simulations are $\left|r_{88}\right|^{2}=0.91,\left|r_{1010}\right|^{2}=0.90$, and $R_{2}>0.998$.

Here, (4) has been applied to pairs of modes that differ by two in order. Somewhat surprisingly, input fields weighted in this way encounter a high reflectivity, even for quite unsymmetric waveguides. The drop in the modal field strength at the core/cover interface with growing $n_{s}$ seemingly compensates for the modal asymmetry when the fields are adjusted to a zero total field at the core/substrate interface. Fig. 9 illustrates the total reflection effect for a nonsymmetric facet. The higher substrate refractive index manifests itself by the more pronounced radiation, the shorter wavelength, and by the slightly longer mode tails in the substrate when compared to the cover region.

\section{CONCLUSION}

Rigorous numerical experiments on multimode reflection at rectangular facets of thick high-contrast slab waveguides led to the following observations.

Single high-order guided modes propagating toward the facet are subject to a large reflectivity, provided the mode angles in the core material are larger than the critical angle for total reflectance at the facet. In that case, the transmitted field consists mainly of two cylindrical waves, originating from the edges of the core. With the shape of the transmission being independent from the input mode, two modes with sufficiently large propagation angle and a difference of two in the mode order can be launched such that the individual transmitted fields interfere destructively; the optical power is then almost entirely reflected into the two input modes. We observed reflectivity levels well above $99 \%$. The effect occurs for properly selected relative input amplitudes if the optical field strength vanishes at the core edges.

While it may be impossible or irrelevant to realize such a configuration directly experimentally, the conditions for full reflectivity, the properly selected relative mode amplitudes, are apparently established automatically at resonance if such a facet with a sufficiently large refractive index contrast is employed as a reflector in a suitably dimensioned resonator. The first evidence for this statement is found in the agreement between the field shapes observed in the core regions next to the facets in Figs. 4 and 9 and the standing wave patterns that appear inside the rectangular cavities of [11] and [12].

\section{ACKNOWLEDGMENT}

The authors would like to thank H. J. W. M. Hoekstra for many fruitful discussions on the subject.

\section{REFERENCES}

[1] C. Vassallo, Optical Waveguide Concepts. Amsterdam, The Netherlands: Elsevier, 1991. 
[2] J. Buus, M. C. Farries, and D. J. Robbins, "Reflectivity of coated and tilted semiconductor facets," IEEE J. Quantum Electron., vol. 27, pp. 1837-1842, June 1991

[3] C. M. Herzinger, C. C. Lu, T. A. DeTemple, and W. C. Chew, "The semiconductor waveguide facet reflectivity problem," IEEE J. Quantum Electron., vol. 29, pp. 2273-2281, Aug. 1993.

[4] J. Xu, D. Yevick, and S. Gallant, "Approximate methods for modal reflectivity at optical waveguide facets," J. Opt. Soc. Amer., vol. 12, no. 4 pp. 725-728, 1995 .

[5] P. Sewell, M. Reed, T. M. Benson, and P. C. Kendall, "Full vector analysis of two-dimensional angled and coated optical waveguide facets," IEEE J. Quantum Electron., vol. 33, pp. 2311-2318, Dec. 1997.

6] O. Vasseur, "Reflectivity of dielectric coatings deposited on the end facet of a weakly guiding fiber," J. Opt. Soc. Amer., vol. 15, no. 1, pp. 77-83, 1998.

[7] A. Vukovic, P. Sewell, T. M. Benson, and P. C. Kendall, "Facet reflectivity of waveguides buried at realistic depth," Proc. SPIE, vol. 3620, pp. 98-108, 1999.

[8] P. Bienstmann, "Rigorous and Efficient Modeling of Wavelength Scale Photonic Components," Ph.D. dissertation, Univ. of Ghent, Ghent, Belgium, 2001

9] P.-A. Besse, J.-S. Gu, and H. Melchior, "Reflectivity minimization of semiconductor laser amplifiers with coated and angled facets considering two-dimensional beam profiles," IEEE J. Quantum Electron., vol. 27, pp. 1830-1836, June 1991.

[10] C. Vassallo, "Finite-difference derivation of the reflectivity at output facets of dielectric waveguides with a highly diverging output beam," J. Opt. Soc. Amer., vol. 15, no. 3, pp. 717-726, 1998.

[11] C. Manolatou, M. J. Khan, S. Fan, P. R. Villeneuve, H. A. Haus, an J. D. Joannopoulos, "Coupling of modes analysis of resonant channel add-drop filters," IEEE J. Quantum Electron., vol. 35, pp. 1322-1331, Sept. 1999.

[12] M. Lohmeyer, "Mode expansion modeling of rectangular integrated optical microresonators," Opt. Quantum Electron., vol. 34, no. 5, pp. $541-557,2002$.
[13] G. Sztefka and H. P. Nolting, "Bidirectional eigenmode propagation for large refractive index steps," IEEE Photon. Technol. Lett., vol. 5, pp. 554-557, May 1993.

[14] M. Lohmeyer and R. Stoffer, "Integrated optical cross strip polarizer concept," Opt. Quantum Electron., vol. 33, no. 4/5, pp. 413-431, 2001

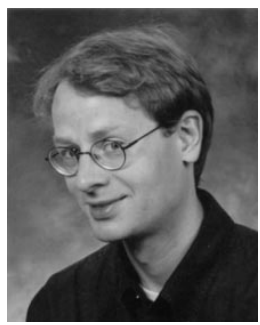

Manfred Hammer (Manfred Lohmeyer until May 2001) studied physics and received the Diplom-Physiker degree from the University of Osnabrück, Germany, in 1995 for work on the numerical analysis of nonreciprocal light propagation in magnetooptic structures in integrated optics. As a Research Assistant at the University, he completed the Ph.D. degree in July 1999, with a thesis titled "Guided waves in rectangular integrated magnetooptic devices."

Since May 2000, he has been a Lecturer in the chair Applied Analysis and Mathematical Physics in the Faculty of Mathematical Sciences, University of Twente, The Netherlands.

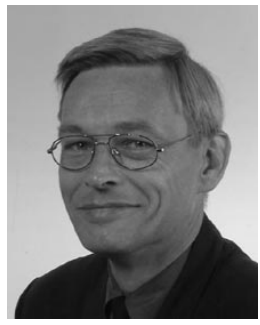

E. van Groesen was born in Breda, The Netherlands, on December 29, 1949. He received the M.Sc. and the Ph.D. degrees in applied mathematics from Technical University of Eindhoven, The Netherlands, in 1973 and 1978 , respectively.

Since 1990, he holds the Chair of Applied Analysis and Mathematical Physics at the University of Twente, The Netherlands. His research interests are nonlinear phenomena and variational methods with application domains fluid dynamics and mathematical optics. 